\title{
Semi-active damping optimization of vibrational systems using the parametric dominant pole algorithm
}

\author{
Peter Benner ${ }^{1, * *}$, Patrick Kürschner ${ }^{1, * * *}$, Zoran Tomljanović ${ }^{2, *}$, and Ninoslav Truhar ${ }^{2, \dagger}$ \\ ' Max Planck Institute for Dynamics of Complex Technical Systems, Computational Methods in Systems and Control \\ Theory, Magdeburg, Germany \\ 2 Department of Mathematics, University J.J. Strossmayer in Osijek, 31000 Osijek, Croatia
}

Received 15 July 2014, revised 12 January 2015, accepted 9 April 2015

Published online 5 May 2015

Key words Semi-active damping, dominant poles, vibrational systems, Lyapunov equations, energy minimization. MSC (2010) 74P10, 70J10, 74G65, 70Q05

\begin{abstract}
We consider the problem of determining an optimal semi-active damping of vibrating systems. For this damping optimization we use a minimization criterion based on the impulse response energy of the system. The optimization approach yields a large number of Lyapunov equations which have to be solved. In this work, we propose an optimization approach that works with reduced systems which are generated using the parametric dominant pole algorithm. This optimization process is accelerated with a modal approach while the initial parameters for the parametric dominant pole algorithm are chosen in advance using residual bounds. Our approach calculates a satisfactory approximation of the impulse response energy while providing a significant acceleration of the optimization process. Numerical results illustrate the effectiveness of the proposed algorithm.
\end{abstract}

\section{Introduction}

Consider a vibrational system described by

$$
\begin{aligned}
M \ddot{q}(t)+C_{11} \dot{q}(t)+K q(t) & =B_{2} u(t)+E_{2} w(t), \\
y(t) & =C_{2} \dot{q}(t), \\
z(t) & =H_{1} q(t),
\end{aligned}
$$

where the matrices $M$ and $K$ (called mass and stiffness, respectively) are real, symmetric positive definite matrices of order $n$. The matrices $B_{2} \in \mathbb{R}^{n \times p}$ and $C_{2} \in \mathbb{R}^{p \times n}$ are control and measurement matrices, while $E_{2} \in \mathbb{R}^{n \times 4}$ represents the primary excitation matrix. The internal damping $C_{1}$ is usually taken to be a small multiple of the critical damping denoted by $C_{\text {crit }}$, that is (see, e.g., $[6,7,25]$ ),

$$
C_{u}=\alpha_{c} C_{\text {crit }}, \quad \text { where } \quad C_{\text {crit }}=2 M^{1 / 2} \sqrt{M^{-1 / 2} K M^{-1 / 2}} M^{1 / 2} .
$$

The state variables contained in the coordinate vector $q(t) \in \mathbb{R}^{n}$ represent displacements and $z(t) \in \mathbb{R}^{s}$ is the performance output which is described by the constant matrix $H_{1} \in \mathbb{R}^{s \times n}$. If one is also interested in the velocities, the output $z(t)$ can include an additional part which corresponds to the velocities, but in this paper only the states are of interest. The vectors $u(t) \in \mathbb{R}^{p}$ and $w(t) \in \mathbb{R}^{4}$ are the control and primary excitation (i.e. noise) inputs, respectively.

We consider the case of a negative linear feedback corresponding to a linear damper of the form

$$
u(t)=-G y(t)
$$

where $G \in \mathbb{R}^{p \times p}$ is a diagonal matrix $G=\operatorname{diag}\left(g_{1}, g_{2}, \ldots, g_{p}\right)$, called damping matrix. The non-negative entries $g_{i}$ represent the friction coefficients of the corresponding dampers. These coefficients are in the following referred to as gain parameters $g_{i}$ for $i=1, \ldots, p$ and can be constant or variable with time within feasible margins, e.g., $g_{i}(t) \in\left[g_{i}^{-}, g_{i}^{+}\right]$

\footnotetext{
Corresponding author E-mail: ztomljan@mathos.hr. Phone: +38531 224 817. Fax: + 3853122480 l

".mail: benner@mpi-magdeburg.mpg.de

-... E-mail: kuerschner@mpi-magdeburg.mpg.de

E-mail: ntruhar@mathos.hr
} 
for $i=1, \ldots, p$. The internal damping given by (4) with $\alpha_{i}>0$ ensures that (1)-(3) is asymptotically stable which helps during the damping optimization.

By using the control $"$ given in (5) together with the usual assumption $C_{2}=B_{2}^{T}[10]$, we obtain

$$
\begin{aligned}
M \ddot{q}(t)+C \dot{q}(t)+K q(t) & =E_{2} w(t) . \\
z(t) & =H_{1} q(t) .
\end{aligned}
$$

where $C:=C_{11}+B_{2} G B_{3}^{T}$ is symmetric, positive definite such that above system is asymptotically stable. The matrices $B_{2}$ and $G$ constitute the semi-active damping part of the above second order system. More details regarding stability and the given model can be found in $[9,10]$.

The transfer function matrix of (6)-(7), obtained by the Laplace transform, is given by

$$
F(s)=H_{1}\left(s^{2} M+s C+K\right)^{-1} E_{2}, \quad s \in \mathbb{C} .
$$

With the substitutions $x_{1}=q$ and $x_{2}=\dot{q}$ we obtain a representation of our vibrating system as the first order differential equation

$$
\begin{aligned}
& \dot{r}(t)=A x(t)+E w(t), \\
& z(t)=H x(t),
\end{aligned}
$$

where

$$
\begin{aligned}
& x=\left[\begin{array}{l}
x_{1} \\
x_{2}
\end{array}\right], A(G)=\left[\begin{array}{cc}
0 & I \\
-M^{-1} K & -M^{-1}\left(C_{n}+B_{2} G B_{2}^{I}\right)
\end{array}\right] . \quad E=\left[\begin{array}{c}
0 \\
M^{-1} E_{2}
\end{array}\right] . \\
& \text { and } \quad H=\left[\begin{array}{ll}
H_{1} & 0
\end{array}\right] .
\end{aligned}
$$

The main problem is to determine "the hest" damping matrix $G$ which will minimize the output $z$ under the influence of the input $w$.

Our aim will be the construction of an efficient algorithm that optimizes the damping matrix $G$. Then one can improve the performance by a switching strategy which will ensure at least the same performance. The switching strategy can be in an on-off mode, which arises in problems of a semi-active damping, see $[9,10]$ for more details.

In damping optimization one can consider different criteria (see, e.g., [10]). For our purposes we will consider the impulse response energy which corresponds to the system's $\mathcal{H}_{2}$-norm. In the frequency domain we define a cost function $J_{2}$ in terms of the transfer function matrix (8) via

$$
J_{2}=\int_{-\infty}^{+\infty} \operatorname{tr}\left(F(j \omega)^{*} F(j \omega)\right) \mathrm{d} \omega .
$$

Using the standard theory (see, e.g. $[10,11,28]$ ), it can be shown that $J_{2}$ can be expressed in the form

$$
J_{2}=\operatorname{tr}\left(E^{T} P E\right) \text {, }
$$

which is much more convenient for numerical computations. Here, $E$ is as in (10) and $P$ is the solution of the following Lyapunov equation

$$
A^{T} P+P A=-H^{T} H
$$

with the matrices $A, H$ given in (10)-(11).

This leads to an optimization problem of determining the optimal matrix $G$ such that

$$
\operatorname{tr}\left(E^{T} P E\right) \rightarrow \min .
$$

Damping optimization using the given criterion requires solving the Lyapunov equation (14) numerous times, which in general could be inefficient, as well as memory and time consuming. This is even the case when state of the art numerical methods for large-scale Lyapunov equations (see, e.g., the surveys [5,24]) are employed. Thus, our aim is to introduce an approach which calculates approximations of the second-order system such that the optimization process is significantly accelerated.

There exist a number of different methods for calculating the approximated system. A review of different methods for this dimension reduction can be found in $[1,2,4]$. In [6.7] the approximation is based on dimension reduction of second-order systems for optimizing a passive damping. It tums out that these approaches are not feasible for semi-active damping optimization due to the different structure in the right-hand side of the corresponding Lyapunov equations and different problem setting. Thus. we were not able to derive an algorithm based on [6.7] which is efficient and accurate 
enough. In this paper we use another strategy based on an interpolatory eigenvalue based approach from [23] in order to optimize the semi-active damping efficiently. This approach uses a small number of selected eigenvalues and -vectors corresponding to the second order system (6)-(7). Since these can be computed efficiently by the dominant pole algorithm $[18,21]$, this can be a computational advantage compared to other model reduction techniques for dynamical systems. Moreover, for parameter dependent systems, it enables to reuse certain data for different gain parameters.

In the next section, we will at first briefly describe the main ideas of the class of dominant pole algorithms, including also the parametric dominant pole algorithm which we plan to use in damping optimization. Afterwards, a strategy for determining good initial parameters for the parametric dominant pole algorithm, and a complete gain optimization routine using the proposed reduction techniques, are presented. Section 3 illustrates the effectiveness of the method by virtue of numerical examples and Sect. 4 concludes.

\section{Parametric dominant pole algorithm for damping optimization}

Since our optimization criterion can be written in terms of the transfer function (12), our aim is to have a good approximation of it. For this purpose we will use an approximation obtained with the dominant pole algorithm. The transfer function (8) can be expressed as a function of eigenvalues of the corresponding quadratic eigenvalue problem. If the algebraic multiplicity equals the geometric multiplicity for all eigenvalues, the transfer function (8) can be represented as

$$
F(s)=\sum_{i=1}^{2 n} \frac{R_{i}}{s-\lambda_{i}}
$$

with the residues

$$
R_{i}=\left(H_{1} x_{i}\right)\left(y_{i}^{*} E_{2}\right) \lambda_{i} \in 2^{5 * q} .
$$

Here, $\lambda_{i} \in \mathcal{L}, x_{i}, y_{i} \in \mathcal{I}^{\prime \prime} \backslash\{0\}$ are, respectively, eigenvalues, right and left eigenvectors of the quadratic eigenvalue problem

$$
\left(\lambda_{i}^{2} M+\lambda_{i} C+K\right) x_{i}=0, \quad y_{i}^{*}\left(\lambda_{i}^{2} M+\lambda_{i} C+K\right)=0, \quad i=1, \ldots 2 n .
$$

Hence, eigenvalues of (17) are poles of (8). For a proper approximation of the transfer function $F(s)$ from (16) we introduce the concept of dominant poles. There are different definitions of dominant poles, and we will use one which is well suited from our point of view regarding semi-active damping optimization.

Definition 2.1 (Dominant Poles [15.16, 18,21]). For the transfer function $F(s)$ in (16), a pole $\lambda_{i}$ will be called dominant if

$$
\frac{\left\|R_{i}\right\|}{\operatorname{Re}\left(\lambda_{i}\right)}>\frac{\left\|R_{j}\right\|}{\operatorname{Re}\left(\lambda_{j}\right)} \quad \forall j \neq i .
$$

Regarding the underlying eigenvalue problem (17), $x_{i}, y_{i}$ are then also referred to as dominant right and left eigenvectors. The $k$-most dominant poles are the $k$ poles (eigenvalues) $\lambda_{i}$ with the largest values of $\frac{\left\|R_{i}\right\|}{R_{i}\left(\lambda_{i}\right)}$.

First we would like to emphasize that the importance of the input-output behavior is, on the one hand, included in the residue $\left\|R_{i}\right\|$. On the other hand, if some pole is close to the imaginary axis (real part of the pole is small) it can have a greater influence to the energy, but if $\left\|R_{i}\right\|$ is very small (due to the input and output matrices) this influence might not be that important as one sees from the fraction $\frac{\left\|R_{i}\right\|}{\mathcal{R}_{\mathrm{e}}\left(\lambda_{i}\right)}$. Furthermore, in the Bode magnitude plot $(\omega,\|F(i \omega)\|)$ it can be observed that peaks occur at frequencies close to the imaginary parts of the dominant poles of $F(s)$ [20] which also explains the importance of the dominant poles.

In order to have a good approximation of the transfer function (8) we will approximate it by its dominant terms. That is, using the first $k$ dominant poles, the transfer function is approximated as

$$
F(s) \approx F_{k}(s)=\sum_{i=1}^{k} \frac{R_{i}}{s-\lambda_{i}} .
$$

The approximation $F_{k}(s)$ is also referred to as transfer function modal equivalent [16, Sect. IV] of $F(s)$ corresponding to the eigentriples $\left(\lambda_{i}, x_{i}, y_{i}\right), i=1, \ldots, k$. The reduced transfer function $F_{k}(s)$ belongs to the reduced order model

$$
\begin{aligned}
Y^{*} M X \ddot{q}_{k}(t)+Y^{*} C X \dot{q}_{k}(t)+Y^{*} K X q_{k}(t) & =Y^{*} E_{2} w(t), \\
z(t) & =H_{1} X q_{k}(t) .
\end{aligned}
$$


where $q(t) \approx X q_{k}(t)$ and $X, Y \in C^{n \cdot k}$ span the eigenspaces associated with the $k$ selected dominant eigenvalues of the quadratic eigenvalue problem. Since the reduced system (19) is defined by matrices of the dimension $k$, we will call the parameter $k$ reduced dimension. It is important to recall that in our setting $M, C$ and $K$ are real, symmetric, positive definite matrices. Hence, if $x_{i}$ is a right eigenvector for an eigenvalue $\lambda_{i}$, then $y_{i}=\bar{x}_{i}$ is a left eigenvector for $\lambda_{i}$ and vice-versa. Moreover, because the eigenvalues and eigenvectors occur in complex conjugate pairs, the space spanned by the right eigenvectors is equal to the space spanned by the left eigenvectors, and, thus $Y=X$ can be chosen. For the reduced system consequently we require only the right eigenvectors and the original system (6)-(7) can be approximated by

$$
\begin{aligned}
X^{*} M X \ddot{q}_{k}(t)+X^{*} C X \dot{q}_{k}(t)+X^{*} K X q_{k}(t) & =X^{*} E_{2} w(t), \\
z(t) & =H_{1} X q_{k}(t) .
\end{aligned}
$$

The calculation of dominant poles can be performed by using an efficient structure-exploiting, specially tailored eigenvalue algorithm which will be described in the next subsection.

\subsection{Subspace accelerated dominant pole algorithm for second order systems}

In this section we will summarize an algorithm based on approaches from [18,21] which calculates the dominant poles and the corresponding eigenvectors utilizing the second-order structure of the multi-input multi-output system. The algorithm we are interested in is the subspace accelerated quadratic MIMO dominant pole algorithm (SAQMDP) [18,21] which is a generalization of the MIMO dominant pole algorithm (SAMDP) [15.16] to second order systems. The main ingredient in the derivation of dominant pole algorithms is the observation that, for any transfer function matrix $F(s)$, the function

$$
f(s):=\left(\sigma_{\max }(F(s))\right)^{-1} \rightarrow 0
$$

as $s$ approaches an eigenvalue of the quadratic eigenvalue problem. The basic idea beyond dominant pole algorithms is then to formally apply a Newton scheme to the above function. This gives a sequence of approximate eigenvectors that converge to the right and left eigenvectors corresponding to a dominant pole of $F(s)$. This iteration can be understood as a modified Rayleigh quotient iteration and further details regarding the convergence behavior can be found in [22]. Storing these approximate eigenvectors obtained in each iteration step in subspaces, i.e., adding the concept of subspace acceleration, yields the subspace accelerated dominant pole algorithm $[19,20]$. Exploiting the second order structure leads to SAQMDP [18,21]. Some modifications to these original algorithms based on the observations above provided by the symmetry and realness of $M, C, K$ can be included which yields Algorithm 1. We briefly describe the main steps of Algorithm 1 in the following.

Step 2: The Newton process applied to (21) requires the computation of the right and left singular vectors $\boldsymbol{\|}$, $z$ corresponding to the largest singular value of $F\left(s^{(1)}\right)$, see $[18,19]$.

Step 4: The linear systems of equations are intrinsically also a part of the Newton step. It is assumed that we are able to solve these large and sparse linear systems by direct solvers.

Step 5-7: These steps belong to the subspace acceleration phase. The solutions $v^{(j)}, w^{(j)}$ of (22) are used as orthogonal expansions for the spaces spanned by $V$ and $W$. Here we apply similar strategies as proposed in [26] to ensure real matrices $V, W$, i.e., the expansion is carried out using $\left[\mathcal{R} e v^{(j)}, \mathcal{I} m v^{(j i)}\right]$ and $\left[\mathcal{R} e w^{(j)}, \mathcal{I} m w^{(j)}\right]$. A Petrov-Galerkin projection is performed onto the second order system. The dominant pole $\theta^{(j)}$ and its corresponding eigenvectors $\tilde{x}^{(j)}, \tilde{y}^{(j)}$ of the resulting small system in Step 6 can be computed by direct methods for eigenvalue problems. In our implementation, the quadeig routine [13] worked satisfactory for this task. In Step 7 approximate eigenvectors of the large-scale, $n$-dimensional problem are computed as well as the corresponding residuals which enable an accuracy assessment of the current approximate eigenpair.

Step 8-18: If the norms of the right and left eigenvalue residuals $\left\|r_{R}^{(j)}\right\|,\left\|r_{L}^{(j)}\right\|$ are small enough, we accepted the eigenpair approximation $\left(\theta^{(i)}, x^{(j)}\right)$. In Step 10 we detect the best eigenvector approximation because we learned before that $x_{i}=\bar{y}_{i}$. The eigenvector and eigenvalue matrices $X, S$ are augmented appropriately, also employing techniques from [26] in case of complex data. In order to prevent detecting this found eigenpair again by the algorithm. it is deflated from the process. There exist different deflation approaches and we point towards the relevant literature [19.21] for details. After that. new direction vectors $u$. z corresponding to $F\left(\theta^{(i)}\right)$ are computed as in Step 2. 


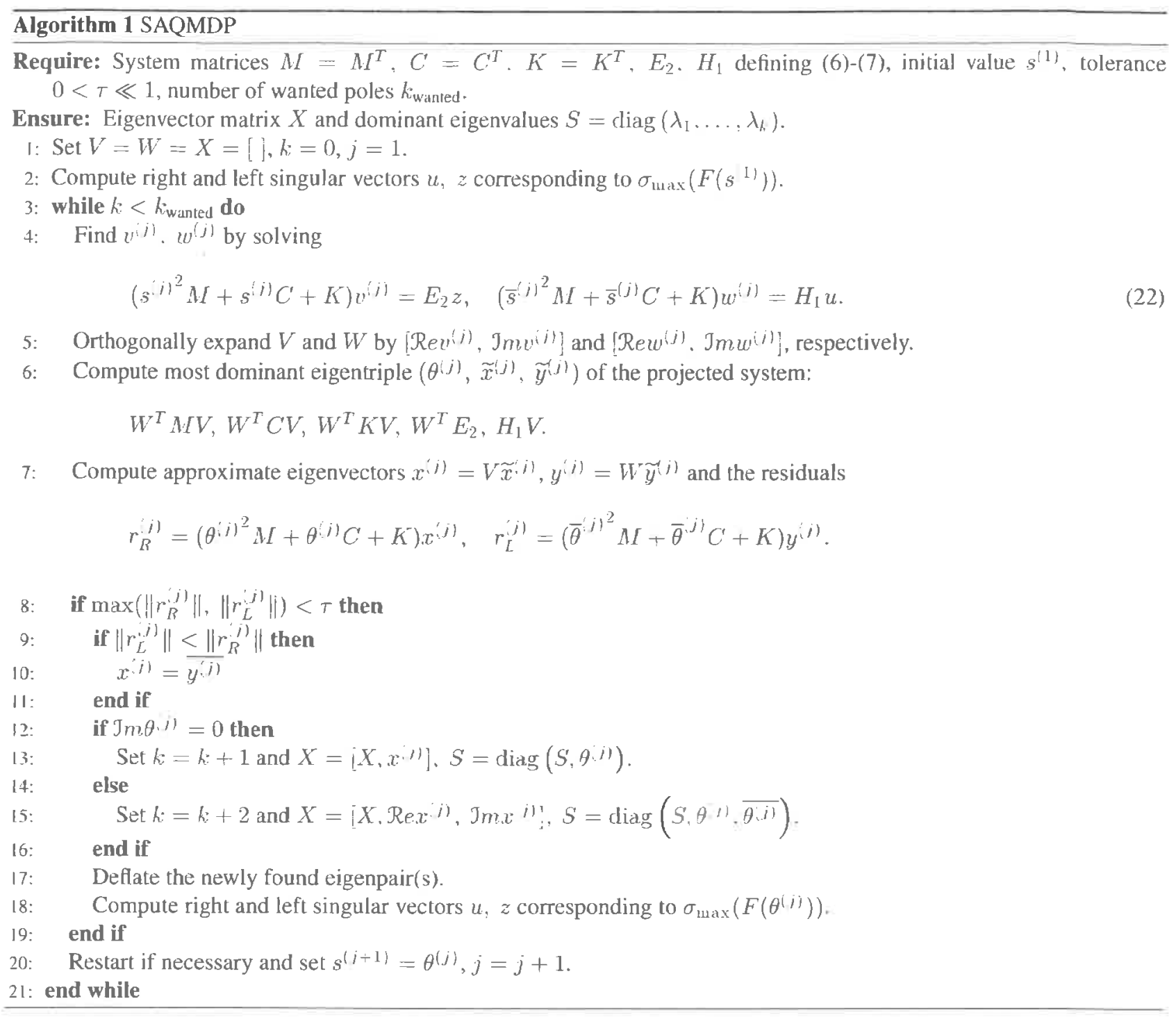

Step 20: A restart is performed if necessary to prevent that the column dimensions of $V$ and $W$ become too large. performed.

Although for the systems considered here only right eigenvector approximations are required, SAQMDP still works with right and left basis matrices $V$ and $W$ because in general $v^{(j)} \neq \overline{w^{(j)}}$ unless $E_{2}=H_{1}^{T}$. Only in the limit it holds that $v^{(j)} \rightarrow x$ and $w^{(j)} \rightarrow \bar{x}$.

\subsection{Parametric dominant pole algorithm}

Our aim is to obtain an accurate reduced system for various gains since we would like to optimize these gains for the corresponding configuration of the system matrices. In the given parametric system (6), the matrix $C=C(g)$ depends on the semi-active damping parameters $g=\left(g_{1}, g_{2}, \ldots, g_{p}\right)$. The main idea in the parametric dominant pole algorithm is to compute approximations acquired with the dominant pole algorithm for a small number of selected parameters and to obtain an 'interpolatory' reduced model regarding other values for $g$.

In [23] it is shown that with such an interpolation based approach, the original and reduced model have the same behavior near the dominant poles and the parametric reduced model obtained with this parametric dominant pole algorithm satisfies a Hermite interpolation property. 
Proposition 2.2 [23, Theorem 2]. Let $x \in \operatorname{span}\{X\}, y \in \operatorname{span}\{Y\}$ for an eigentriple $(\lambda, x, y)$ of (17) for a given g. Moreover, let $F(s), \widehat{F}(s)$ denote the original and reduced transfer function matrices of (6)-(7), where $\widehat{F}(s)$ is obtained using (19). Assume that $\lambda$ is simple and that $y^{*} x \neq 0, y^{*} x-|\lambda|^{2} M^{-1} y^{\times} B_{2} B_{2}^{T} x \neq 0$. Then it holds

$$
\lim _{s \rightarrow \lambda}|s-\lambda|^{2} \frac{\partial F}{\partial g}=\lim _{r \rightarrow \AA}|s-\lambda|^{2} \frac{\partial \widehat{F}}{\partial g} .
$$

P r o of. The proof can be carried out exactly as for [23. Theorem 2] by rewriting the second order system (6)-(7) into a first order system (9) and exploiting the structure of the matrices $A, E, H$.

The interpolation property is a desirable and important property for gain optimization since a reduced model which is a good approximation of the original model for various values of gains $g$ is required. Hence, using the parametric dominant pole algorithm can be advantageous over other model reduction approaches that do not have the Hermite interpolation property.

There are different implementations for the interpolatory dominant pole algorithm (for more details see [23]) and we will summarize an approach based on SAQMDP for the calculation of the reduced system. An algorithm called continuation SAQMDP is illustrated in Algorithm 2 and computes reduced models that will enable an efficient semi-active damping optimization.

The selected values of parameters for which we will calculate dominant poles will be called initial parameters and are denoted by $g^{\prime \prime}, g^{(2)}, \ldots, g^{\left({ }^{\prime \prime \prime}\right)}$ with $g^{(i)}=\left(g_{1}^{(i)}, g_{2}^{(i)}, \ldots, g_{p}^{(i)}\right)$. For these values the reduced transfer function approximates the original transfer function in the sense of $(18)$ and the reduced model satisfies interpolation properties as shown in $[23]$.

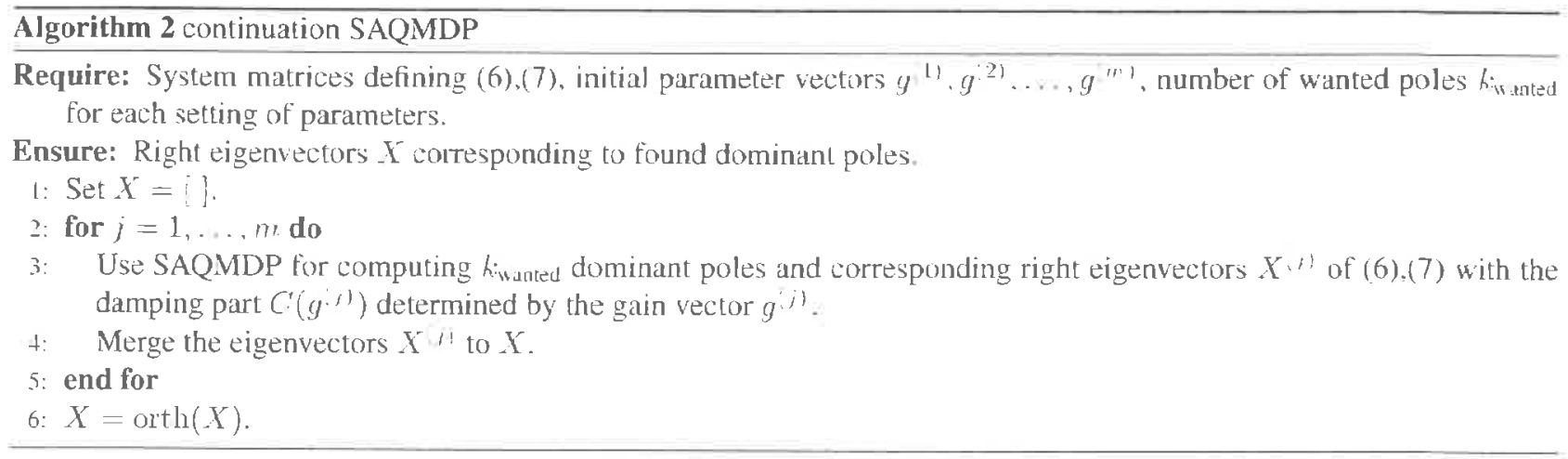

In Step 6 of Algorithm 2 we introduce an additional orthogonalization. There, a clever orthogonalization routine should be employed to ensure that numerically linearly dependent columns in $X$ are discarded and the matrix $X$ has full column rank such that the reduced system will have regular coefficient matrices. It is noteworthy that in principle, also several other model reduction techniques [8] can be used in Step 3 to provide the matrices $X^{(j)}$ for a given $g^{(j)}$. However, as we are interested in the dominant poles of the system's transfer function, the application of SAQMDP is chosen here. Note that in order to improve the convergence to dominant poles in Step 3, we can use the most dominant pole from the previous run of SAQMDP as new initial frequency value $s^{(1)}$. Using the obtained eigenvectors collected in the matrix $X$ from Algorithm 2, the reduced system can be formed as in (20).

It is important to point out that the mass, damping and stiffness matrices of the reduced system are again symmetric, positive definite and thus, the reduced system is also asymptotically stable.

Next, we draw special attention to one particular setting of initial parameters, namely the case with initial gain $g^{(1 ;}=0$.

\subsection{Modal approximation}

In the case of a zero gain $(g=0)$, the system is only damped by the internal damping term. In order to simplify the analysis of this particular case, we transform the system using the so-called modal coordinates. For that purpose let $\Phi=\left[\phi_{1}, \ldots \phi_{n}\right]$ denote the nonsingular matrix which simultaneously diagonalizes $M$ and $K$, i.e.,

$$
\Phi^{T} K \Phi=\Omega^{2}=\operatorname{diag}\left(\omega_{1}^{2} \ldots, \omega_{n}^{2}\right) \text { and } \Phi^{T} M \Phi=l .
$$

where $0<\omega_{1} \leq \omega_{2} \leq \ldots \leq \omega_{n}$. The columns of the matrix $\Phi$ (usually called modal matrix) are the eigenvectors of the undamped system $M \ddot{q}+K q=0$ and the positive numbers $\omega_{1}$. $\omega_{2} \ldots \ldots \omega_{n}$ are the associated eigenvalues which are referred 
to as undamped eigenfrequencies. Furthermore, the matrix $\Phi$ also diagonalizes the internal damping as $\Phi^{T} C_{u} \Phi=\alpha \Omega$ with $\alpha=2 \alpha_{c}$.

By using (23) and substituting $q(t)=\Phi \widehat{q}(t)$, we obtain from (6)-(7) the vibrational system:

$$
\begin{aligned}
\ddot{q}(t)+\left(\alpha \Omega+\Phi^{T} B_{2} G B_{2}^{T} \Phi\right) \hat{q}(t)+\Omega^{2} \widehat{q}(t) & =\Phi^{T} E_{2} w(t), \\
z(t) & =H_{1} \Phi \widehat{q}(t) .
\end{aligned}
$$

As in the introduction, with $\widehat{x}_{1}=\Omega \widehat{q}$ and $\widehat{x}_{2}=\hat{q}$, the associated first order system is

$$
\begin{aligned}
\hat{x}(t) & =\widehat{A} \widehat{x}(t)+\widehat{E} w(t), \\
z(t) & =\widehat{H} \widehat{x}(t),
\end{aligned}
$$

where

$$
\widehat{x}:=\left[\begin{array}{l}
\widehat{x}_{1} \\
\widehat{x}_{2}
\end{array}\right], \quad \widehat{A}:=\left[\begin{array}{cc}
0 & \Omega \\
-\Omega & -\alpha \Omega-\Phi^{T} B_{2} G B_{2}^{T} \Phi
\end{array}\right], \quad \widehat{E}:=\left[\begin{array}{c}
0 \\
\Phi^{T} E_{2}
\end{array}\right],
$$

and $\widehat{H}:=\left[H_{1} \Phi \Omega^{-1} 0\right]$. The minimization criterion (15) leads to the Lyapunov equation

$$
\widehat{A}^{T} \widehat{P}+\widehat{P} \widehat{A}=-\widehat{H}^{T} \widehat{H} \text {. }
$$

A Lyapunov equation of similar structure was investigated in the papers [6,7], where the authors employ dimension reduction techniques in order to efficiently approximate its solution.

By formulating the criterion (15) in terms of the solution of the Lyapunov equation (27), a direct calculation shows that

$$
P=U^{*} \widehat{P} U, \quad \text { where } U=\left[\begin{array}{cc}
\Phi \Omega^{-1} & 0 \\
0 & \Phi
\end{array}\right] \text {, }
$$

where $P$ and $\widehat{P}$ solve (14) and (27), respectively. Furthermore, if we uniformly partition $\widehat{P}, E$ as

$$
\widehat{P}=\left[\begin{array}{ll}
P_{11} & P_{12} \\
P_{21} & P_{22}
\end{array}\right], \quad E=\left[\begin{array}{c}
0 \\
M^{-1} E_{22}
\end{array}\right] .
$$

the impulse response energy (15) is

$$
J_{2}=\operatorname{tr}\left(E^{T} P E\right)=\operatorname{tr}\left(E^{T} U^{*} \widehat{P} U E\right)=\operatorname{tr}\left(E_{2}^{T} \Phi P_{22} \Phi^{T} E_{2}\right) .
$$

Now, we will construct an approximation for the given impulse response energy using eigenvectors $\phi_{i}$ of the undamped system. The eigenvectors of the system corresponding to the zero gain are given in the matrix $\Phi$, i.e. the matrix $X$ from (20) corresponding to $g=0$ contains the columns of $\Phi$. Approaches that use undamped eigenvectors for forming the reduced system are usually called modal approximations $[7,12,27]$.

In order to decide which undamped eigenvectors should be chosen, we consider the structure of criterion (15) of the system in modal coordinates (24)-(25). Here, the right-hand side of the Lyapunov equation (27) has a special structure which strongly influences the solution. The main question is: in terms of the criterion (15), which undamped eigenvectors should be chosen in this particular case?

For the answer we consider the structure of the right-hand side in (27):

$$
\widehat{H}^{T} \widehat{H}=\left[\begin{array}{cc}
\Omega^{-1} \Phi^{T} H_{1}^{T} H_{1} \Phi \Omega^{-1} & 0 \\
0 & 0
\end{array}\right] \text {. }
$$

Furthermore, we obtain

$$
\Phi \Omega^{-1}=\left[\begin{array}{llll}
\frac{1}{\omega_{1}} \phi_{1} & \frac{1}{\omega_{2}} \phi_{2} & \cdots & \frac{1}{\omega_{n}} \phi_{n}
\end{array}\right]
$$

and a direct multiplication reveals that the upper diagonal block has the following elements

$$
\left(\Omega^{-1} \Phi^{T} H_{1}^{T} H_{1} \Phi \Omega^{-1}\right)_{i j}=\frac{1}{\omega_{i} \omega_{j}} \phi_{i}^{T} H_{1}^{T} H_{1} \phi_{j}
$$

These elements are quadratic in $\frac{1}{\omega_{r}}$ and since the $\omega_{i}$ are ordered increasingly, for maintaining the elements largest (by magnitude) in the matrix $\widehat{H}^{T} \widehat{H}$, we select the columns of $\Phi$ such that, in the right hand side of Lyapunov equation (27). the $r$ largest numbers are maintained. Altogether this means that in (20)

$$
X=\Phi(: .1: r)
$$


is chosen, i.e., the upper diagonal $r \times r$ block of the right hand-side of (27). In the system equation with zero gain, the eigenvalues of the corresponding eigenvalue problem $\left(\lambda^{2} M+\lambda \alpha_{t} C_{\text {trit }}+K\right) x=0$ have the same eigenvectors as the corresponding undamped eigenvaluc problem $\left(\lambda^{2} M+K\right) x=0$. Furthermore, it can be shown that eigenvectors which correspond to the smallest undamped eigenvalues (i.e. smallest $\omega_{i}$ ) are the same eigenvectors that correspond to the eigenvalues closest to the imaginary axis of the eigenvalue problem $\left(\lambda^{2} M+\lambda \alpha_{c} C_{\text {crit }}+K\right) x=0$. This means that truncating with the eigenvectors that correspond to the smallest undamped eigenvalues is similar to a truncation with eigenvectors which correspond to eigenvalues closest to the imaginary axis of the zero gain eigenvalue problem. This also reflects the importance of the poles close to the imaginary axis, especially for the case of small gains.

For the selection $X=\Phi(:, 1: r)$, the reduced system (20) becomes

$$
\begin{aligned}
\ddot{q}_{r}(t)+\Phi(:, 1: r)^{T} C \Phi(:, 1: r) \dot{q}_{r}(t)+\Omega_{r} q_{r}(t) & =\Phi(: 1: r)^{T} E_{2} w(t), \\
z(t) & =H_{1} \Phi(:, 1: r) q_{r}(t),
\end{aligned}
$$

where $\Omega_{r}=\operatorname{diag} \omega_{1}, \omega_{2}, \ldots, \omega_{r}$. The corresponding Lyapunov equation is

$$
\widetilde{A}^{T} Q+Q \tilde{A}=-\tilde{H}^{T} \tilde{H}
$$

with

$$
\widetilde{A}=\left[\begin{array}{cc}
0 & \Omega_{r} \\
-\Omega_{r} & -\alpha \Omega_{r}-X^{r} B_{2} G B_{2}^{T} X
\end{array}\right], \quad \widetilde{H}=\left[\begin{array}{cc}
H_{1} X \Omega_{r}^{-1} & 0
\end{array}\right] .
$$

Hence, the impulse response energy $J_{2}$ can be approximated by

$$
J_{2} \approx \operatorname{tr}\left(E_{2}^{T} X Q_{22} X^{r} E_{2}\right)
$$

with $Q_{22}$ being the lower diagonal block of the solution $Q$ of (29).

Modal approximation plays an important role in engineering applications. In our case it allows us to conclude, directly from criterion (15), which undamped eigenvectors are important for a gain equal to zero. Furthermore. modal approximation can be easily incorporated into our approach which uses continuation SAQMDP by setting the initial parameter vector $g$ ' equal to zero. In the optimization procedure this requires insignificant computations since these eigenvectors have to be compuled only once in the process which can be achieved efficiently using iterative eigenvalue solvers for generalized eigenvalue problems. The usage of such modal approximations in our approach will be discussed in the next subsection.

\subsection{Gain optimization}

In the numerical examples we will illustrate that a few different values of parameters $g^{(1)}, g^{(2)}, \ldots, g^{(m)}$ are often sufficient to obtain satisfactory approximations of the optimal gains. Furthermore, by changing the gains by small or even moderate values, the same eigenvectors can provide good approximations of the eigenspaces. Thus, the dimension of the reduced system is usually much smaller than the full dimension. In this section we will show how this can be determined in advance. Furthermore, in many applications we observe that, even with eigenvectors that correspond to the zero initial gain, we can obtain sufficiently accurate approximations of the original system in the sense of gain optinization. Thus, we include the eigenspace corresponding to the zero gain in the first approximation.

We would like to efficiently determine initial parameters $g^{(1)}, g^{(2)}, \ldots, g^{(m)}$ and at this moment it is not clear how one can choose the initial gains. Thus, we will derive corresponding residual bounds which will provide additional information. In this setting, the main question is: if we have computed an (approximate) eigenspace for the gain $g^{(i)}$, what should he the next gain $g^{(i+1)}$ for which it is required to compute a new eigenspace?

First, recall that the gain $g^{(i)}=\left(g_{1}, g_{2}, \ldots, g_{p}\right)$ determines the damping part $C\left(g^{(i)}\right)=C_{11}+B_{2} G B_{2}^{T}$ where $G=$ $\operatorname{diag} g_{1} . g_{2} \ldots, g_{p}$. Let us assume that for the gain $g^{(i)}$, using Algorithm 1 , we have calculated $k$ dominant right eigenvectors $x^{(1)}, x^{(2)} \ldots \ldots x^{(k)}$ and corresponding eigenvalues $\theta^{(1)}, \theta^{(2)}, \ldots, \theta^{(k)}$ for a residual tolerance $\tau$ :

$$
\left\|\left(\theta^{(j)^{2}} M+\theta^{(j)}\left(C_{u}+B_{2} \operatorname{diag}\left(g_{1}, g_{2}, \ldots, g_{p}\right) B_{2}^{T}\right)+K\right) x^{(j)}\right\|<\tau, \quad \forall j=1, \ldots k .
$$

The residual corresponding to these approximate eigenpairs for the system with gain $g^{(i)}+\Delta g^{(i)}$ is, using (31),

$$
\begin{aligned}
\|\left(\theta^{(j)^{2}} M\right. & \left.+\theta^{(j)} C\left(g^{(i)}+\Delta g^{(i)}\right)+K\right) x^{(j)} \| \\
& =\left\|\left(\theta^{(j)^{2}} M+\theta^{(j \cdot} \cdot\left(C_{\|}+B_{2} \operatorname{diag}\left(g_{1}+\delta g_{1}, g_{2}+\delta g_{2}, \ldots, g_{r}+\delta g_{p}\right) B_{2}^{T}\right)+K\right) x^{(j)}\right\| \\
& <\tau+\left\|\theta^{(j)} B_{2} \operatorname{diag}\left(\delta g_{1}, \delta g_{2}, \ldots \delta g_{p}\right) B_{2}^{T} x^{(j)}\right\| \\
& \leq \tau+\left\|\theta^{(j,} B_{2}\right\|\left\|\operatorname{diag}\left(\delta g_{1}, \delta g_{2} \ldots . \delta g_{p}\right)\right\|\left\|B_{2}^{T} x^{(j \cdot}\right\| . \quad \forall j=1, \ldots, k .
\end{aligned}
$$


Our aim is to determine for which perturbation $\Delta g^{i i}=\left(\delta g_{1}, \delta g_{2}, \ldots, \delta g_{p}\right)$ the same subspace will be also good up to fixed new tolerance $\nu$.

In general,

$$
\tau \ll\left\|\left(\theta^{(j)^{2}} M+\theta^{\prime j)} C\left(g^{(i)}+\Delta g^{(i)}\right)+K\right) x^{\prime \prime \prime}\right\| \leq u, \quad \forall j=1, \ldots, k
$$

because the approximate eigenpairs $\left.\left(\theta^{i} i\right), x^{i j}\right)$ correspond to the system with gain $g^{(i)}$. We would like to determine $\Delta g^{(i)}$ such that the same eigenvectors are also good for $g^{i)}+\Delta g^{(i)}$ for a prescribed tolerance $v$. Since $\tau \ll v$ we neglect $\tau$ in the formula above and we have that

$$
\begin{aligned}
\left\|\left(H^{(j)^{2}} M+\theta^{(i)} C\left(g^{(i)}+\Delta g^{(i)}\right)+K\right) x^{(j)}\right\| & <\tau+\left\|\theta^{j)} B_{2}\right\|\|\| \Delta g^{(i)}\|\|\left\|B_{2}^{T} x^{(i)}\right\| \\
& \lesssim\left\|\theta^{(j)} B_{2}\right\|\left\|\Delta g^{(i)}\right\|\left\|B_{2}^{T} x^{(j)}\right\| \leq v, \quad \forall j=1, \ldots, k .
\end{aligned}
$$

Thus, for $\tau \ll v$ if

$$
\left\|\Delta g^{, i)}\right\| \leq \frac{v}{\left\|\theta^{\prime \prime \prime} B_{2}\right\|\left\|B_{2}^{T} x^{(i)}\right\|}, \quad \forall j=1, \ldots, k .
$$

holds for given tolerance $v$ the eigenspace obtained for $g^{(i)}$ will also give proper approximation for $g^{(i)}+\Delta g^{(i)}$. A measure that takes all computed approximate eigenpairs into account can be formulated by using the mean average over the upper bounds for all eigenpairs. The upper bound for an acceptable change of $g^{(i)}$ associated to all eigenpairs is thus determined by

$$
r_{i}:=\frac{1}{k} \sum_{j=1}^{k} \frac{v}{\left\|\theta^{i} j B_{2}\right\|\left\|B_{2}^{T} x^{j i j}\right\|}
$$

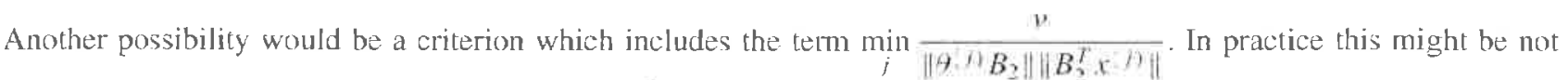
appropriate since very few indices $j$ where $\frac{\|}{\left\|B_{3}\right\| B^{l} \mathrm{x}\|\|}$ is small while in the other cases this is quite large.

To incorporate the bound obtained into the algorithm for the gain optimization, we introduce

$$
K\left(g^{(j)}, r_{j}\right):=\left\{g:\left\|g-g^{(j)}\right\| \leq r_{j}\right\},
$$

where $r_{j}$ is defined in (32).

Note that, if we assume that all dampers have the same gains $\left(G=g I_{p}\right)$, then we can obtain a shatper bound for $\Delta g^{(i)} \in \mathbb{R}$ as

$$
\left|\Delta g^{(i)}\right| \leq \frac{v}{\left\|\theta^{(j)} B_{2} B_{2}^{T} x^{(i)}\right\|}, \quad \forall j=1, \ldots, k
$$

and use

$$
r_{i}=\frac{1}{k} \sum_{j=1}^{k} \frac{v}{\left\|\theta^{(j)} B_{2} B_{2}^{T} x^{(j)}\right\|}
$$

in this case. The efficiency of our gain optimization algorithm can be improved by using the above bounds.

We would like to emphasize that we determine the initial gains $g^{(2)}, g^{(3)}, \ldots, g^{(m)}$ adaptively using the above mentioned bounds. In particular, we start the optimization procedure with modal approximation, i.e. the initial gain at beginning $g^{(1)}=0$. Then, in the optimization procedure for the current initial gains $g^{(1)}, g^{(2)}, \ldots, g^{(j)}$ if next gain approximation $g \in \bigcup_{i=1}^{j} K\left(g^{(i)}, r_{i}\right)$, we do not update the subspace. Otherwise, we add this gain $g$ to the set of initial gains (i.e. $g^{(j+1 i}=g$ ) and update the reduced model for which we can now calculate the function value $J_{2}$. During the evaluation for the current initial gains $g^{(1)}, g^{(2)}, \ldots, g^{(j)}$, if $g \in \bigcup_{i=1}^{j} K\left(g^{(i)}, r_{i}\right)$, then the eigenvalue subspace obtained so far is good enough for this gain also, and we calculate an approximation of the function value $J_{2}$ using the current reduced model. If this is not the case, we add this gain $g$ to the set of initial gains (i.e. $g^{(j+1)}=g$ ) and update the reduced model ( $X$ is enriched by new eigenvectors) from which we can now calculate the function value $J_{2}$.

Algorithm 3 illustrates this approach for the gain optimization. In order to clarify Algorithm 3 note that: in Step I we set the initial gain and in step 2 we run the optimization procedure. In Steps 3 to 12 an approximation of $J_{2}$ is calculated and the initial gains are updated. 


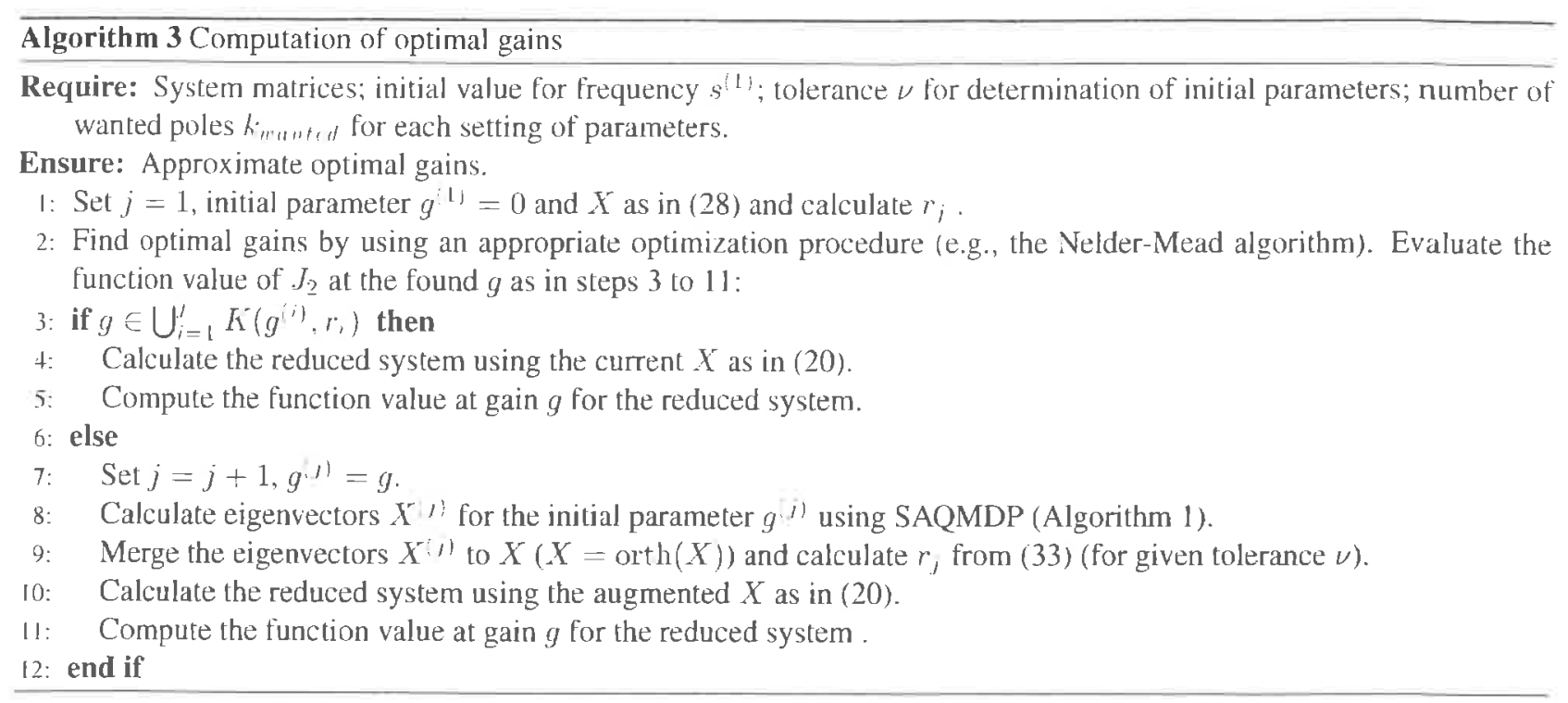

Note that, in our setting we optimize over nonnegative variables, and thus, we have to modify the usage of the NelderMead algorithm. In particular, at the evaluation of the objective $J_{2}$ we prescribe that this function at a point with at least one negative variable is equal to a very large constant (e.g., we take a constant that is significantly larger than the function value at the zero gain). This ensures that the Nelder-Mead algorithm will give an optimal value that is nonnegative. Moreover, in order to have relevant function values during the optimization procedure, if we increase the order of the reduced system. we also update all function values with respect to the changed reduced systems.

The calculation of the eigenvectors corresponding to the zero initial gain should be carried out only once for the whole optimization procedure. Furthermore, including the eigenvectors of the undamped system additionally stabilizes the optimization procedure. In particular, due to the occasional slow convergence of the SAQMDP for some configurations. we might end up with a very small reduced dimension which yields a reduced system which is not accurate enough. With the zero initial gain we, however, always end up with reduced dimensions that are greater or equal to the number of wanted poles.

\section{Numerical experiments}

In this section we illustrate the efficiency of the introduced approximation techniques. For that purpose we will compare the new approach with the optimization without reduction. In both approaches the Lyapunov equations are solved by the Hammarling's algorithm [14] implemented in the MATLAB ${ }^{\mathrm{F}}$ function 1 yapchol based on the SLICOT routines SB03OD and SG03BD [3].

In these examples, the computations have been carried out on a server with 4 Intel Xeon @2.67 GHz CPUs with 8 cores per CPU and 1 TB RAM. Results were calculated in MATLAB Version 7.11.0.584 (R2010b) 64-bit.

Example 3.1. We will consider an $n$-mass oscillator or oscillator ladder which describes the mechanical system of $n$ masses and $n+1$ springs (see e.g. [7]). The mathematical model is given by (1)-(3), where the mass and stiffness matrices are

$$
\begin{aligned}
& M=\operatorname{diag}\left(m_{1}, m_{2}, \ldots, m_{n}\right) . \\
& K=\left(\begin{array}{ccccc}
k_{1}+k_{2} & -k_{2} & & \\
-k_{2} & k_{2}+k_{3} & -k_{3} & & \\
& \ddots & \ddots & \ddots & \\
& & -k_{n-1} & k_{n-1}+k_{n} & -k_{n} \\
& & & -k_{n} & k_{n}+k_{n+1}
\end{array}\right) .
\end{aligned}
$$

We will consider the following configuration for the mass and stiffness values:

$$
n=1800: \quad k_{i}=2 . \quad \forall i: \quad m_{j}= \begin{cases}1500-2 i . & i=1 \ldots 600 \\ 600-i / 2 . & i=601 \ldots 999 . \\ i-800 . & i=1000 \ldots \ldots\end{cases}
$$


The internal damping is given by the damping matrix (4) with $\alpha_{t}=0.002$. For simplicity $G$ is a multiple of the identity, i.e., matrix $G=\operatorname{diag} g, g, \ldots, g \in \mathbb{R} p p$ with $p=6$. The control matrix is given by

$$
B_{2}=\left[e_{j} e_{i+1} e_{j+10} e_{j+11} e_{i+20} e_{j+21}\right] \text {, }
$$

where $1 \leq j+21 \leq n$ and $e_{j}$ is the $j$ th canonical vector. Here, we have introduced an index $j$ since we will consider different configurations for $B_{2}$. Furthermore, we are interested in the states in the first third of the n-mass oscillator that correspond to the smallest masses, that is, we consider 40 states starting with the 580 th state:

$$
z(t)=\left[q_{580}(t) q_{581}(t) \ldots q 619(t)\right]^{T} .
$$

Hence, $H_{1} \in \mathbb{P}^{10 / n}$ with

$$
H_{1}(1: 40,580: 619)=I_{40<10}
$$

and all other entries are equal to zero. Since we consider gain optimization for different primary excitation matrices, we define $E_{2} \in \mathbb{R}^{\prime \prime} \times 30$ depending on index $k \geq 1$. The primaty excitation is applied to 30 consecutive masses where the first mass is determined by the index $k$ :

$$
E_{2}(k: k+29,1: 30)=I_{30} \times 30 \text {. }
$$

We compare the approximation of the impulse response energy $J_{2}$ for different damper positions and different primary excitations. i.e., the following configurations of $(k, j)$ are taken into consideration:

$$
\begin{aligned}
& \text { for } k=30: 200: 1800, \quad j=100: 200: 1800, \\
& \text { exclude indices, where for }(j<600 \& k<600) \mid(j>600 \& k>600) \\
& \text { it holds }|j-500|<|k-500|,
\end{aligned}
$$

with $k$ and $j$ determining $B_{2}$ and $E_{2}$ as given by (34) and (35), respectively. This yields in total 60 different starting configurations for the gain optimization. The following parameters required by Algorithms 1-3 are used:

$$
k_{\text {yrant } e^{\prime}}=72, \quad v=3 \cdot 10^{-4}, \quad \tau=10^{-12} .
$$

The initial value for the frequency $s^{(1)}$ in SAQMDP is taken as the eigenvalue closest to the imaginary axis corresponding to the zero initial gain. For this example this is $-2.505 \cdot 10^{-7}+1.252 \cdot 10^{-4} i$,

Since we consider different configurations (we especially change the primary excitation input, the control matrix) we need to sort these configurations. In order to present relative enrors more clearly, the different configurations are sorted w.r.t. the magnitude of the relative error in the optimal gain. Figure 1 presents the relative errors of the optinal gain calculated with and without dimension reduction by Algorithm 3. The circles denote the relative error $\left|g^{0}-g\right| / g$, where $g$ and $g^{0}$ denote the optimal gain calculated with and without dimension reduction, respectively. Similarly, triangles denote the relative errors w.r.t. the approximation of the impulse response energy $J_{2}$, where in the case without dimension reduction, $J_{2}$ was calculated at the exact optimal gains.

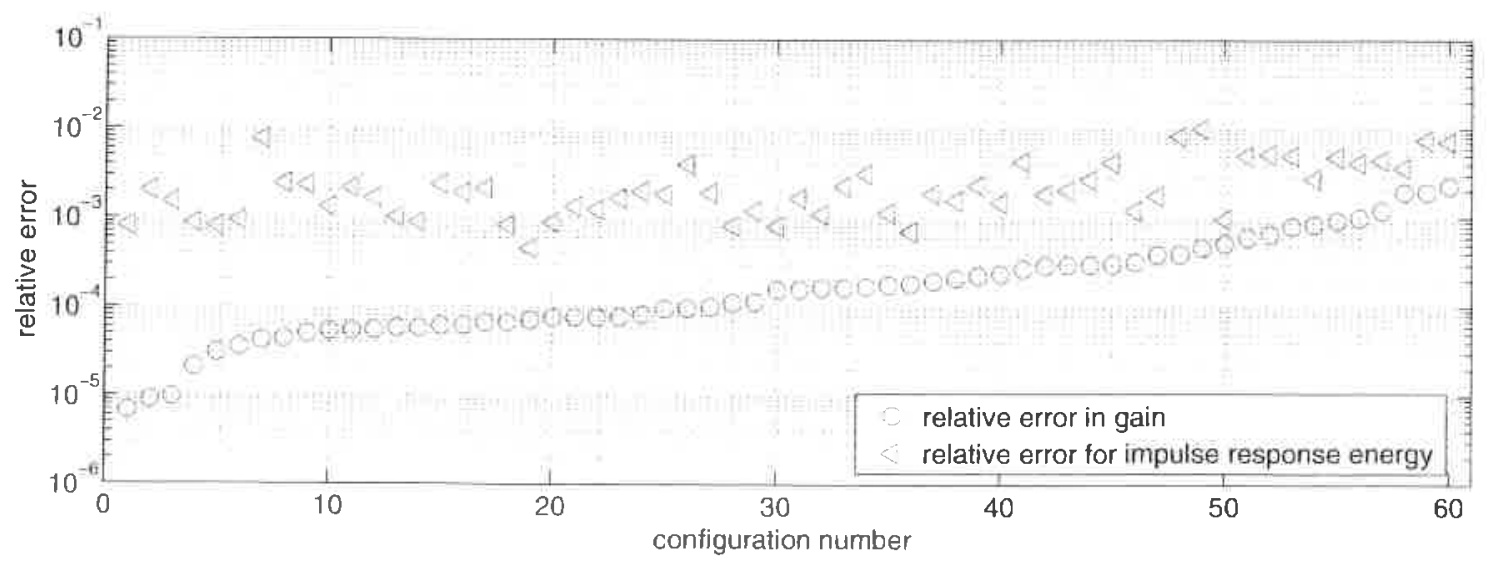

Fig. 1 Relative errors for the gain and the energy $J_{2}$. 

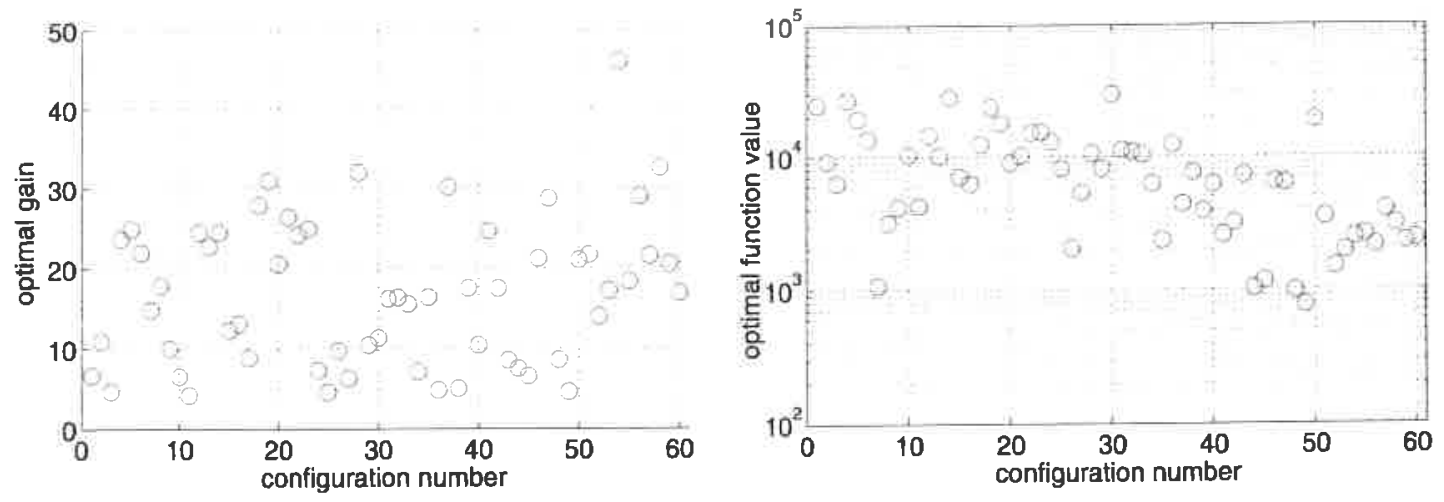

Fig. 2 Magnitudes of the optimal gains and impulse response energies.

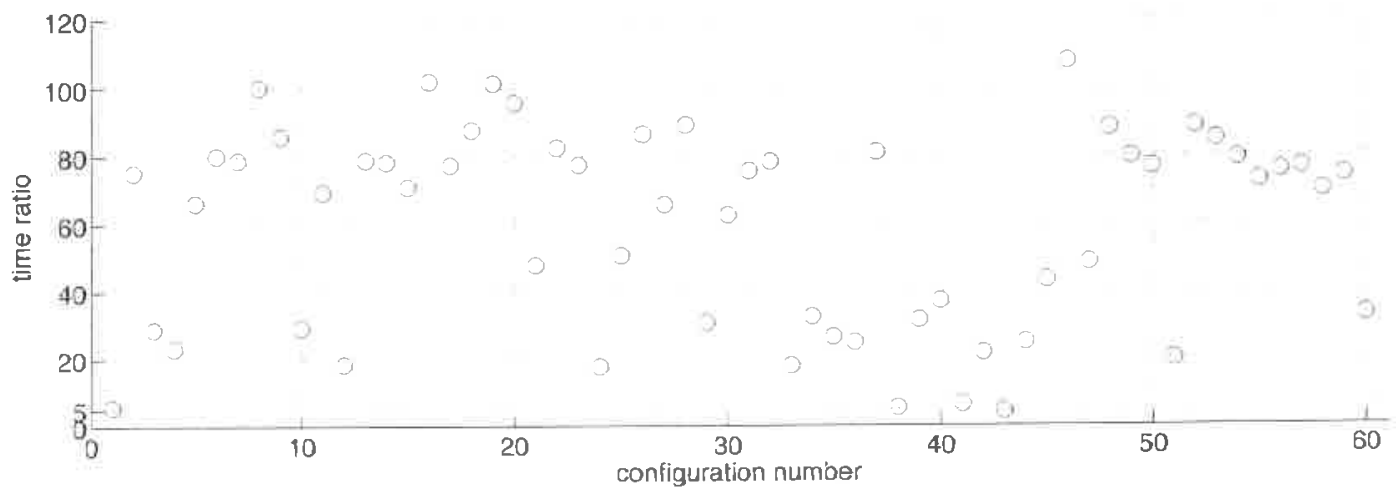

Fig. 3 Time ratio between exact and approximation based approach.

Recall that all gains are constant and equal, thus we can apply Nelder-Mead's method (see e.g. [17]) implemented in the MATLAB function Eminsearch. The tolerance for this optimization was $10^{-5}$ and the initial guess was set to 40 . The magnitude of the optimal gains and impulse response energies $J_{2}$ are plotted in Fig. 2. As it can be seen, the optimal gain varies between 4 and 46 .

In Fig. 3 the speed-up in computational time of the optimization process obtained by the new approximation technique is illustrated. More precisely, it shows the ratio of the times required for the gain optimization with and without the novel approximation technique using dimension reduction. Evidently, the new approach requires $\frac{1}{4}$ up to $\frac{1}{107}$ of the computation time required for the unreduced system, amounting to a considerably faster gain optimization process.

Note that in Algorithm 3 we calculated eigenvectors corresponding to $g=0$ and additional gains which are determined using the residual bound from Sect. 2.4. Altogether, in the optimization procedure based on the residual bound, we had to recalculate eigenvectors for one to four additional gains $g$ and, thus, the reduced dimension varies between 90 and 216 (the full dimension is 1800 ). Figure 1 reveals that all relative errors are of order $10^{-2}$ or smaller. For the tolerance $v=3 \cdot 10^{-4}$, the maximal reduced dimension varies between $5 \%$ and $12 \%$ of the original dimension. Figure 4 shows the maximal reduced dimensions for the corresponding configurations.

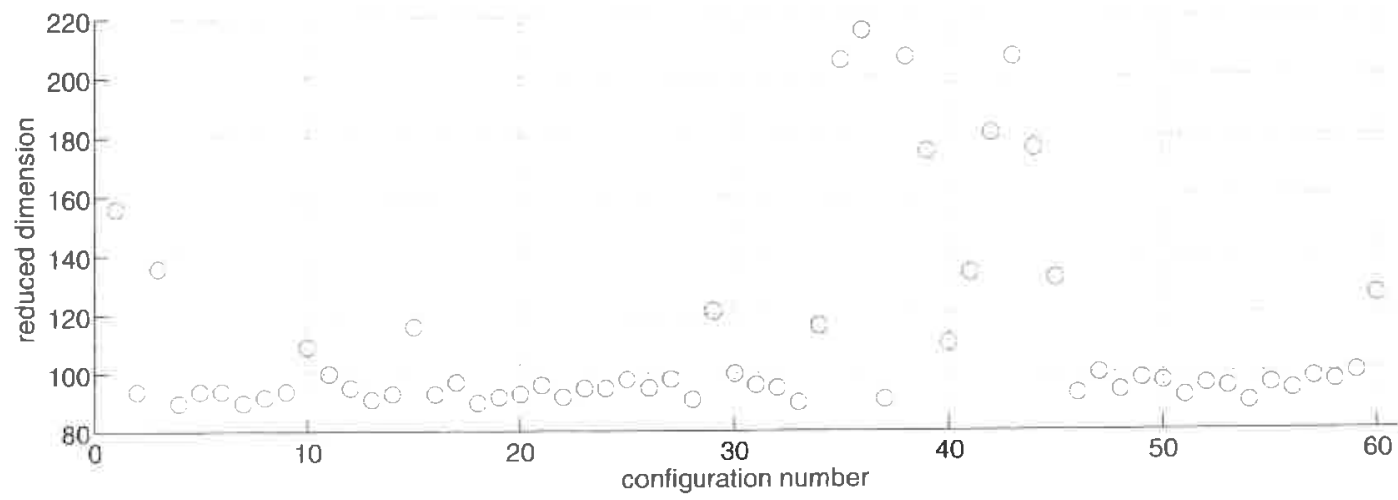

Fig. 4 Reduced dimension. 
In the following example we will compare the quality of the proposed approach on a different mechanical structure. Compared to the previous example we will consider two different gains and compare the results w.r.t. a varying structure of the corresponding control matrices.

Example 3.2. In this example we will consider a mass oscillator with $2 d+1$ masses and $2 d+3$ springs given in [7. Example 2]. This example has two rows of $d$ masses connected with springs where the first row of masses has stiffnesses $k_{1}$ and the second row has stiffnesses $k_{2}$. The first masses on the left edge $\left(m_{1}\right.$ and $\left.m_{d+1}\right)$ are connected to a fixed bound while on the other side of the rows the masses $\left(m_{d}\right.$ and $\left.m_{2 d}\right)$ are connected to a mass $m_{2 d+1}$ with a stiffness $k_{3}$ connected to a fixed bound.

The mass matrix is a diagonal matrix as in the previous example and the stiffness matrix is defined by

$$
K=\left[\begin{array}{ccc}
K_{11} & & -\kappa_{1} \\
& K_{22} & -\kappa_{2} \\
-\kappa_{1}^{T} & -\kappa_{2}^{T} & k_{1}+k_{2}+k_{3}
\end{array}\right] \text {. }
$$

where

$$
K_{i i}=k_{i}\left[\begin{array}{ccccc}
2 & -1 & & & \\
-1 & 2 & -1 & & \\
& \ddots & \ddots & \ddots & \\
& & -1 & 2 & -1 \\
& & & -1 & 2
\end{array}\right], \quad \kappa_{i}=\left[\begin{array}{c}
0 \\
\vdots \\
0 \\
k_{i}
\end{array}\right], \quad i=1,2 .
$$

We will consider the following configuration for the mass and stiffness values:

$$
\begin{aligned}
& d=800, \quad n=2 d+1=1601 ; \quad k_{1}=3, \quad k_{2}=1, \quad k_{3}=2 ; \\
& m_{i}=3.98+0.02 \cdot i \text { for } i=1, \ldots, 600 ; \\
& m_{i}=34-0.03 \cdot i \text { for } i=601, \ldots, 800 ; \\
& m_{i}=23-0.01 \cdot i \text { for } i=801, \ldots, 1600 ; \quad m_{2 d+1}=10 .
\end{aligned}
$$

With this configuration, masses on the first row are smaller on the edges, while on the second row we have masses that decrease in magnitude.

The internal damping is given by the damping matrix (4) with $\alpha_{c}=0.005$.

Here, we are interested in 20 states. In particular, 10 masses on the first row starting from the 301 st to 310 th mass and on the second row starting from the 1201 st to 1210 th mass. Thus, the matrix $H_{1} \in \mathbb{R}^{10 \times n}$ has the following form

$$
H_{1}(1: 10,301: 310)=I_{10 \times 10}, \quad H_{1}(1: 10,1201: 1210)=I_{10 \times 10},
$$

and all other entries are equal to zero.

The primary excitation input acts on the masses closer to the edges of the mechanical structure and the input is stronger on the edges. Thus we define $E_{2} \in \mathbb{R}^{n \times 11}$ with

$$
E_{2}(1: 5,1: 5)=\operatorname{diag}(5,4,3,2,1), \quad E_{2}(801: 805,6: 10)=\operatorname{diag}(5,4,3,2,1), \quad E_{2}(1601,11)=10,
$$

and all other entries are equal to zero.

In this example we will consider two cases regarding the number of different gains. At first, we consider two different gains $g_{1}$ and $g_{2}$ determining $G$, while in the second part of this example we will consider four different gains which renders the optimization procedure even more complicated.

Case I: For the two different gains a diagonal matrix $G=\operatorname{diag}\left(g_{1}, g_{1}, g_{2}, g_{2}\right) \in \mathbb{R}^{p \times p}$ with $p=4$ is used. The control matrices $B_{2} \in \mathbb{R}^{\prime \prime \times 4}, C_{2}=B_{2}^{T}$ are determined with indices $j$ and $k$ such that

$$
B_{2}=\left[\begin{array}{llll}
\left(e_{j}-e_{j+1}\right) & \left(e_{j+10}-e_{j+11}\right) & \left(e_{k}-e_{k+1}\right) & \left(e_{k+10}-e_{k+11}\right)
\end{array}\right] .
$$


Note that by varying the indices $j$ and $k$ we change the geometry of the control matrix. In particular, we consider the following configuration of the indices $j$ and $k$ :

$$
j=50: 100: 800, \quad k=900: 200: 1600
$$

which yields 32 different configurations. This means that the first two columns of $B_{2}$ determine the control on the first row while the third and fourth column determine the control applied to the second row of masses, respectively.

The following parameters required by Algorithms $1-3$ are used:

$$
k_{\text {yumled }}=120, \quad v=5 \cdot 10^{-3}, \quad \tau=10^{-12} .
$$

The initial value for the frequency $s^{\prime \prime}$ in SAQMDP is taken as the eigenvalue closest to the imaginary axis corresponding to the zero initial gain.

Here, we consider two different gains and, like in the previous example, we apply Nelder-Mead's method, but the tolerance for this optimization was set to $10^{-+}$and the initial guess was set to $[500,500]$. Figure 5 presents the relative errors of the optimal gain calculated with and without dimension reduction by Algorithm 3. We consider different configurations regarding the control and in Fig. 5 different configurations $(j, k)$ are sorted w.r.t. the magnitude of the relative enror in the optimal gain. Similarly, triangles denote the relative errors w.r.t. the approximation of the impulse response energy $J_{2}$, where in the case without dimension reduction, $J_{2}$ was calculated at the exact optimal gains. Figure 5 reveals that in this example all relative errors are of order $10^{-1}$ or smaller. Moreover, the reduced dimension varies between 152 and 757 (full dimension is 1601 ) and more additional gains in the optimization process compared to the previous example were required. This is partially expected since damping optimization with different gains is more demanding, but in general this might also depend on the system configuration. We would like to note that also in this example at each configuration our approach was faster. For the considered 32 configurations, the whole optimization process without the approximation technique required 509.61 hours, while with the approximation technique we needed 40.2 hours.

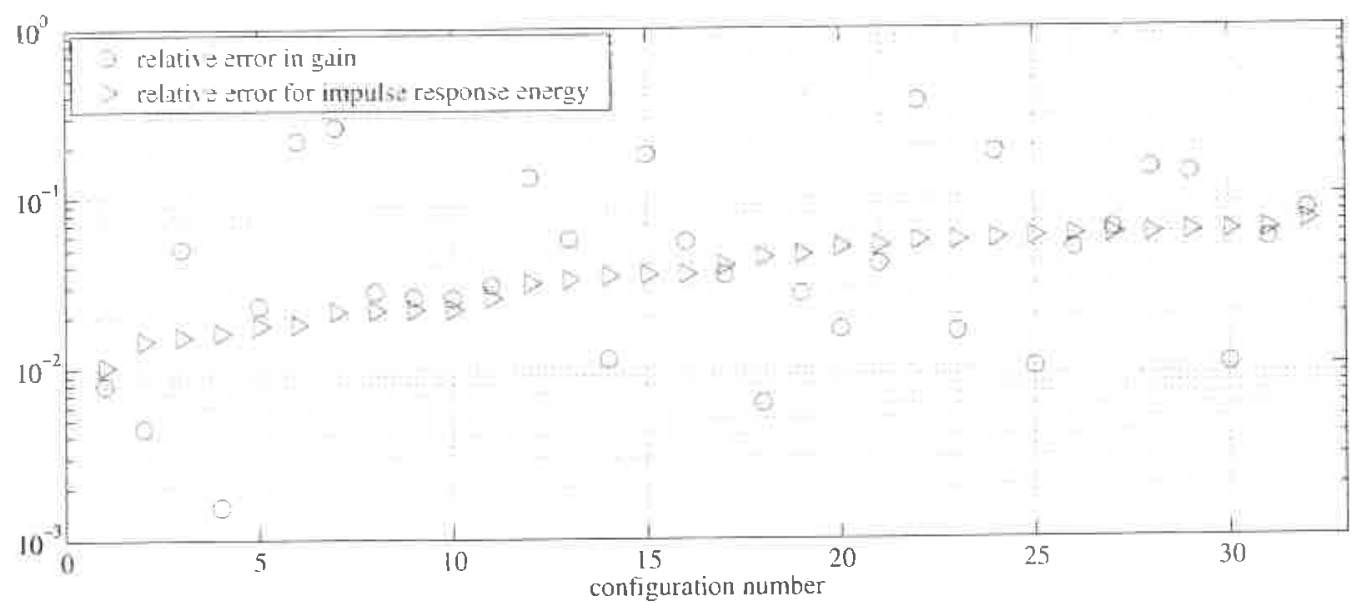

Fig. 5 Relative errors for the gain and the energy $J_{2}$.

Case II: For the four different gains $g_{1}, g_{2}, g_{3}, g_{4}$ we use $G=\operatorname{diag}\left(g_{1}, g_{2}, g_{3}, g_{4}\right) \in \mathbb{R}^{p \times p}$ with $p=4$. Since in this case we will not consider all pairs of indices $(j, k)$, the following table illustrates which particular configurations have been investigated. This means that the system matrices $M, K, E_{2}, H_{1}$ are the same, but the matrix $B_{2}$ given in (36) will be considered for particular settings of the indices $(j, k)$.

As in Case I, here we used the following parameters required by Algorithms 1-3:

$$
k_{\text {unumed }}=120, \quad \nu=1 \cdot 10^{-2} . \quad \tau=10^{-12} .
$$

In Table 1 we summarize the indices $j, k$ determining (36), the function values obtained with and without dimension reduction. the relative errors, the time ratios, as well as the used reduced dimensions for this example.

This example requires many more function evaluations. Moreover, we have observed that one needs more initial gains for the optimization procedure. Thus, one can expect a larger reduced dimensions. This is also shown in Table I where we illustrate the connection of the time ratio with the reduced dimension while ensuring acceptable relative errors in the optimization process. 
Table 1 Numerical results for the case with four difterent gains.

\begin{tabular}{rrrrllcr}
\hline$j$ & & & & \multicolumn{2}{l}{$\begin{array}{l}\text { relative error } \\
\text { in } J_{2}\end{array}$} & $\begin{array}{l}\text { relative error } \\
\text { in gains }\end{array}$ & $\begin{array}{l}\text { reduced } \\
\text { dimension }\end{array}$ \\
\hline 50 & 150 & 252.54 & 244.82 & $3.0575 \cdot 10^{-2}$ & $6.3800 \cdot 10^{-2}$ & 123.77 & 153 \\
150 & 900 & 249.05 & 235.74 & $5.3456 \cdot 10^{-2}$ & $3.7114 \cdot 10^{-2}$ & 94.854 & 159 \\
150 & 1100 & 254.34 & 260.97 & $2.6066 \cdot 10^{-2}$ & $9.8933 \cdot 10^{-2}$ & 11.385 & 538 \\
150 & 1300 & 256.74 & 247.47 & $3.6117 \cdot 10^{-2}$ & $3.8963 \cdot 10^{-2}$ & 89.324 & 174 \\
150 & 1500 & 253.14 & 238.67 & $5.7177 \cdot 10^{-2}$ & $6.1393 \cdot 10^{-1}$ & 108.78 & 155 \\
350 & 900 & 249.48 & 255.50 & $2.4134 \cdot 10^{-2}$ & $6.6207 \cdot 10^{-2}$ & 19.789 & 411 \\
350 & 1100 & 254.77 & 256.95 & $8.5730 \cdot 10^{-3}$ & $4.7500 \cdot 10^{-3}$ & 1.6180 & 1254 \\
350 & 1300 & 257.16 & 263.35 & $2.4062 \cdot 10^{-2}$ & $7.3417 \cdot 10^{-2}$ & 4.6447 & 746 \\
350 & 1500 & 253.63 & 264.64 & $4.3408 \cdot 10^{-2}$ & $8.3629 \cdot 10^{-2}$ & 5.3262 & 596 \\
\hline
\end{tabular}

\section{Conclusions}

In this paper we have considered optimizing a semi-active damping using a criterion based on the impulse response energy. This optimization problem is very demanding due to the numerous Lyapunov equations which have to be solved. We have presented an approach which approximates the impulse response energy by efficiently computing approximations of the transfer function. This is achieved by using the dominant pole algorithm for parametric systems with the initial parameters chosen using eigenvalue residual bounds. Numerical experiments confirm the efficiency of our approach in the sense that the optimization process is considerably accelerated and satisfactory approximations of the optimal gain are obtained.

Acknuwledgement The authors would like to thank the reviewers for the careful reading of the paper and the helpful comments and remarks. This work is supported by the DAAD (PPP Croatia) and the Zeidler-Forschungs-Stittung.

\section{References}

[1] A. Antoulas, Approximation of Large-Scale Dynamical Systems (SLAM Publications, Philadelphia, PA, 2005).

[2] Z. Bai, J. Demmel, J. Dongarra, A. Ruhe, and H. van der Vorst. Templates for the solution of Algebraic Eigenvalue Problems: A Practical Guide (SIAM, Philadelphia, 2000).

[3] P. Benner, V. Mehrmann, V. Sima, S. Van Huffel, and A. Varga, SLICOT - A Subroutine Library in Systems and Control Theory, Appl. Comput. Contr. Sign. Circ. 1, 499-532 (1999).

[4] P. Benner, V. Mehrmann, and D. Sorensen. Dimension Reduction of Large-Scale Systems (Lecture Notes in Computational Science and Engineering, Springer-Verlag, Berlin/Heidelberg, Germany, 2005).

[5] P. Benner and J. Saak, Numerical solution of large and sparse continuous time algebraic matrix Riccati and Lyapunov equations: a state of the an survey, GAMM Mitteilungen 36(1). 32-52 (2013).

[6] P. Benner, Z. Tomljanović, and N. Truhar, Dimension reduction for damping optimization in linear vibrating systems, Z. Angew. Math. Mech. 91(3). 179-19l (2011), DOI: 10.1002/zamm.201000077.

[7] P. Benner, Z. Tomljanović, and N. Truhar, Optimal damping of selected eigenfrequencies using dimension reduction, Numer. Linear Algebr. Appl. 20(1), I-17 (2013), DOI: 10.1002/nla.833.

[8] P. Benner, S. Gugercin, and K. Willcox, A Survey of Model Reduction Methods for Parametric Systems, Max Planck Institute Magdeburg Preprint MPIMD/13-14. August 2013, Available from http://www.mpi-magdeburg.mpg.de/preprints/.

[9] F. Blanchini. D. Casagrande. P. Gardonio, and S. Miani, On Optimal Damping of Vibrating Structures. in: Proceedings of the 18th IFAC World Congress, Milan, Italy, (September 2011).

[10] F. Blanchini. D. Casagrande. P. Gardonio, and S. Miani, Constant and switching gains in semi-active damping of vibrating structures, Int. J. Control 85(12), I $886-1897$ (2012).

[1I] J. B. Burl, Linear Optimal Control: $\mathcal{H}_{2}$ and $\mathcal{H}_{\sim}$ Methods. Ist edition (Addison-Wesley Longman Publishing Co., Inc., Boston. MA, USA, 1998).

[12] W. Gawronski, Advanced Structural Dynamics and Active Control of Structures (Springer. New York, USA, 2004).

[13] S. Hammarling, C. J. Munro, and F. Tisseur. An algorithm for the complete solution of quadratic eigenvalue problems, ACM Trans. Math. Softw. 39(3). 18:1-18:19(2013).

[14] S. J. Hammarling. Numerical solution of the stable. nonnegative definite Lyapunov equation. IMA Journal of Numerical Analysis 2(3), 303-323 (1982). 
[15] N. Martins, L. Lima, and H. Pinto, Computing dominant poles of power system transfer functions, IEEE Trans. Power Syst. 11(1), $162-170(1996)$.

[16] N. Martins and P. Quintao, Computing dominant poles of power system multivariable transfer functions, IEEE Trans. Power Syst. $18(1), 152-159(2003)$.

[17] J. A. Nelder and R. Mead, A simplex method for function minimization, Comput. J. 7, 308-313 (1965).

[18] J. Rommes, Methods for eigenvalue problems with applications in model order reduction, PhD thesis, Universiteit Utrecht, 2007.

[19] J. Rommes and N. Martins, Efficient Computation of Multivariable Transfer Function Dominant Poles Using Subspace Acceleration, IEEE Trans. Power Syst. 21(4), 1471-1483 (2006).

[20] J. Rommes and N. Martins, Efficient computation of transfer function dominant poles using subspace acceleration, IEEE Trans. Power Syst. 21(3), 1218-1226 (2006).

[21] J. Rommes and N. Martins, Computing transfer function dominant poles of large-scale second-order dynamical systems, SIAM J. Sci. Comput. 30(4), 2137-2157 (2008).

[22] J. Rommes and G. L. G. Sleijpen, Convergence of the dominant pole algorithm and Rayleigh quotient iteration 30(1), 346-363 (2008).

[23] M. Saadvandi, K. Meerbergen, and W. Desmet, Parametric dominant pole algorithm for parametric model order reduction, J. Comput. Appl. Math. 259(A), 259-280 (2014).

[24] V. Simoncini, Computational methods for linear matrix equations, Available at http://www.dm.unibo.it/simoncin/, March 20 I3.

[25] N. Truhar and K. Veselić, An efficient method for estimating the optimal dampers' viscosity for linear vibrating systems using Lyapunov equation, SIAM J. Matrix Anal. Appl. 31(1), 18-39 (2009).

[26] T. van Noorden and J. Rommes, Computing a partial generalized real Schur form using the Jacobi--Davidson method, Numer. Linear Algebr. 14(3), 197-215 (2007).

[27] K. Veselić, Damped Oscillations of Linear Systems (Springer Lecture Notes in Mathematics, Springer-Verlag. Berlin, 201 I).

[28] K. Zhou, J. Doyle, and K. Glower, Robust and Optimal Control (Upper Saddle River, New Jersey: Prentice Hall, 1996). 

